\title{
Total elbow arthroplasty for active primary tuberculosis of the elbow: a curious case of misdiagnosis
}

\author{
Radhakrishnan Pattu, Girinivasan Chellamuthu, Kumar Sellappan, Kamalanathan Chendrayan \\ Department of Orthopedics, Government Mohan Kumaramangalam Medical College Hospital, Salem, India
}

\begin{abstract}
The incidence of musculoskeletal tuberculosis (TB) is on the rise due to the current Acquired Immunodeficiency Syndrome (AIDS) pandemic. Spine is the most common osseous site, followed by other joints. TB identified in the elbow accounts for $2 \%-5 \%$ of skeletal TB cases, which are secondary to pulmonary TB. Primary elbow TB is rare. We report a case of primary TB of the elbow which had a negative synovial biopsy. A 46-year-old right-hand dominant female patient with chronic pain and disability of the right elbow was diagnosed with chronic non-specific arthritis based on an arthroscopic synovial biopsy. The case was diagnosed retrospectively as active TB from bone cuts post total elbow arthroplasty. Anti-tuberculosis treatment (ATT) was given postoperatively for 12 months. The patient reported good functional outcomes at 3 years of follow-up. Such atypical presentations of osteoarticular TB are challenging to diagnose. Therefore, particularly in endemic areas, clinicians should be careful before excluding such a diagnosis even after a negative biopsy. Further research should investigate whether active TB of small joints such as the elbow can be treated with ATT, and early arthroplasty should be a focus of this research.
\end{abstract}

Keywords: Arthroplasty; Elbow; Tuberculosis, osteoarticular; Biopsy

Tuberculosis (TB) is an important global health problem. TB cases can be pulmonary or extrapulmonary. Extrapulmonary TB accounts for $20 \%$ to $40 \%$ of cases and musculoskeletal TB accounts for 35\% of extrapulmonary TB cases. Due to the Acquired Immunodeficiency Syndrome (AIDS) pandemic the incidence of musculoskeletal TB is on the rise. Sites that are involved with this type of TB include the spine (Pott disease; $50 \%$ of cases), pelvis (12\%), hip and femur (10\%), knee and tibia (10\%), ribs (7\%), ankle or shoulder (2\%), elbow or wrist (2\%), and multiple sites (3\%) [1]. TB that involves the elbow joint is rare and is almost always secondary to pulmonary TB. Primary TB of the elbow is rare [2]. We report a case of primary TB of the elbow that was missed initially and diagnosed retrospectively as active TB from bone cuts post total elbow arthroplasty (TEA). To the best of our knowledge, this is the first case of TEA reported in active advanced TB of the elbow.

\section{CASE REPORT}

Approval for this study was waived because this study is a case report of a single patient. The patient provided consent for participation and publication.

A 46-year-old right-hand dominant female patient presented with complaints of chronic pain and disability of the right elbow that had persisted for the past 5 years. The patient was Indian by nationality. The pain was low-intensity and non-radiating but

Received: May 13, $2021 \quad$ Revised: July 24, $2021 \quad$ Accepted: July 26, 2021

Correspondence to: Radhakrishnan Pattu

Department of Orthopedics, Government Mohan Kumaramangalam Medical College Hospital, Salem, Tamil Nadu 636001, India

Tel: +91-8667087047, E-mail: arthrork7@gmail.com, ORCID: https://orcid.org/0000-0002-0526-1117

Financial support: None.

Conflict of interest: None.

Copyright@ 2022 Korean Shoulder and Elbow Society.

This is an Open Access article distributed under the terms of the Creative Commons Attribution Non-Commercial License (http://creativecommons.org/licenses/by-nc/4.0/) which permits unrestricted non-commercial use, distribution, and reproduction in any medium, provided the original work is properly cited. 
had progressively increased for the past 6 months. The patient had no history of trauma. There was no history of loss of weight or loss of appetite. The patient denied contact history with TB patients and she was not immunocompromised. Joint line tenderness was present, on examination. Elbow movements were from $0^{\circ}$ to $20^{\circ}$, painful in the available range, and restricted by severe pain afterward. The supination-pronation arc was full but painful. The movements were accompanied by joint crepitus. A plain radiograph demonstrated loss of joint space with the destruction of articular ends of the distal humerus, radial head, and proximal ulna (Fig. 1). The chest radiograph was normal. Blood investigations were within normal limits except for an elevated erythrocyte sedimentation rate $(40 \mathrm{~mm} / \mathrm{hr})$ and C-reactive protein $(14 \mathrm{mg} / \mathrm{L})$. The tuberculin skin test was negative. An arthroscopic synovial biopsy was performed, which revealed chronic non-granulomatous inflammation, ruling out TB. The synovial culture showed no bacterial growth. Therefore, we decided to proceed with TEA to increase mobility and reduce joint pain.

\section{Operative Technique}

Under general anesthesia, the patient was positioned in the left lateral position. Through the posterior approach, the triceps was exposed and retracted laterally. The ulnar nerve was exposed and secured. The distal humerus cut was made just above the olecranon fossa. The radial head was excised above the annular ligament attachment. A subarticular L-shaped cut of the proximal ulna was made to protect the triceps and brachialis attachments. The medullary canals of the ulna and humerus were reamed with
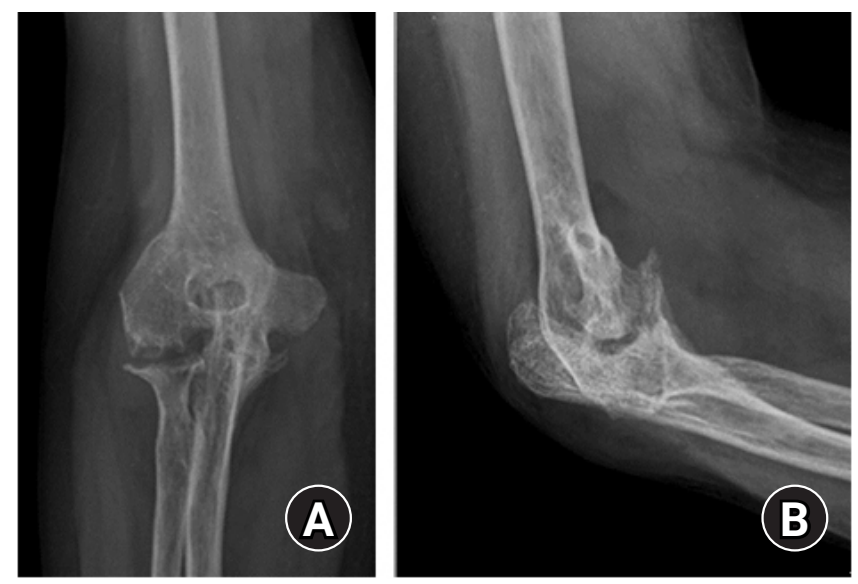

Fig. 1. Plain radiograph demonstrated loss of joint space with the destruction of articular ends of the distal humerus, radial head, and proximal ulna. (A) Anteroposterior radiograph on the right showing loss of joint space and arthritis. (B) Lateral radiograph on the left showing destruction of articular ends of the distal humerus, radial head, and proximal ulna. respective harpoon-shaped reamers. The ulnar and humeral components of the Baksi prosthesis [3] were fixed with bone cement, and the hinge components were assembled with the main screw, and then the locking screw was applied. Fig. 2 shows different components of the slopy hinge Baksi prosthesis that were assembled as a single unit. Fig. 3 shows the assembled prosthesis. Postoperatively, the limb was splinted with $90^{\circ}$ of flexion for seven days after which mobilization was started. Fig. 4 shows the immediate postoperative X-ray.

The resected bone ends were sent for biopsy, which revealed an active TB infection. Thus, a diagnosis of primary active TB of the elbow was made retrospectively. The patient was given anti-tuberculosis treatment (ATT) for 12 months. At the 3-year follow-up timepoint, the patient reported pain-free movement of $0^{\circ}$ to $110^{\circ}$ and a stable joint. Fig. 5 shows the follow-up X-ray at 3 years after the procedure. Fig. 6 demonstrates the range of movements of the affected elbow.

\section{DISCUSSION}

This case report describes a rare presentation of osteoarticular TB which was diagnosed retrospectively. Treatment of TB of the elbow, like all joint TB, is primarily by ATT along with surgeries

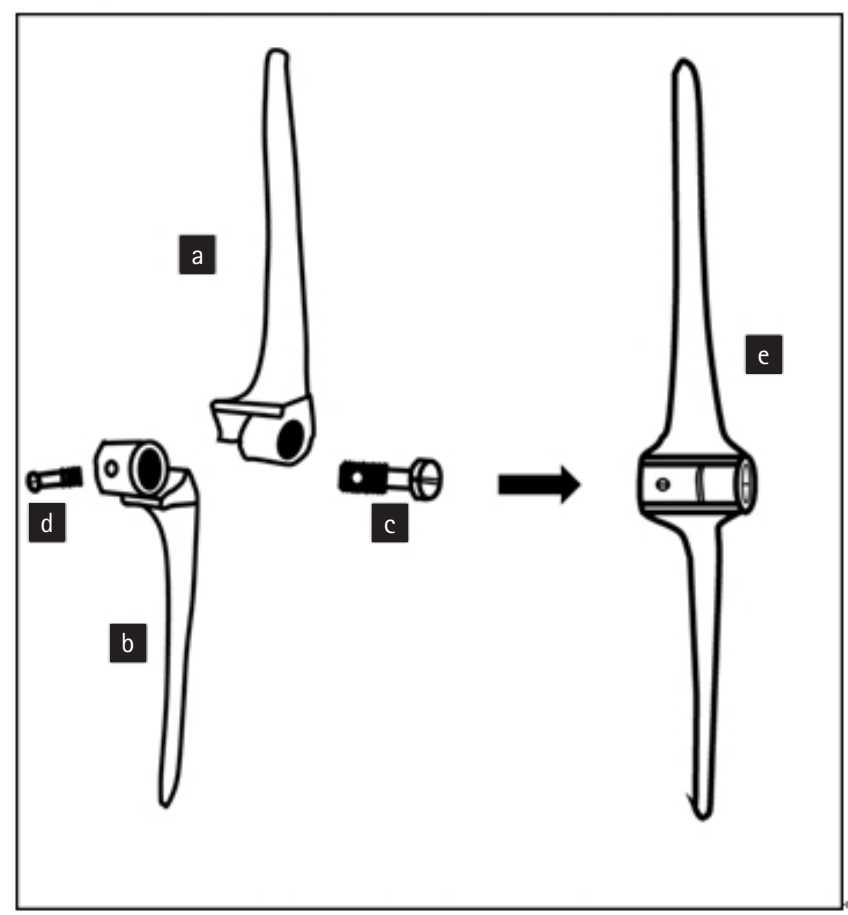

Fig. 2. Line diagram showing the assembly of different components of the Baksi prosthesis. a, humeral component; b, ulnar component; c, main screw; d, additional locking screw; and e, fully assembled unit. 


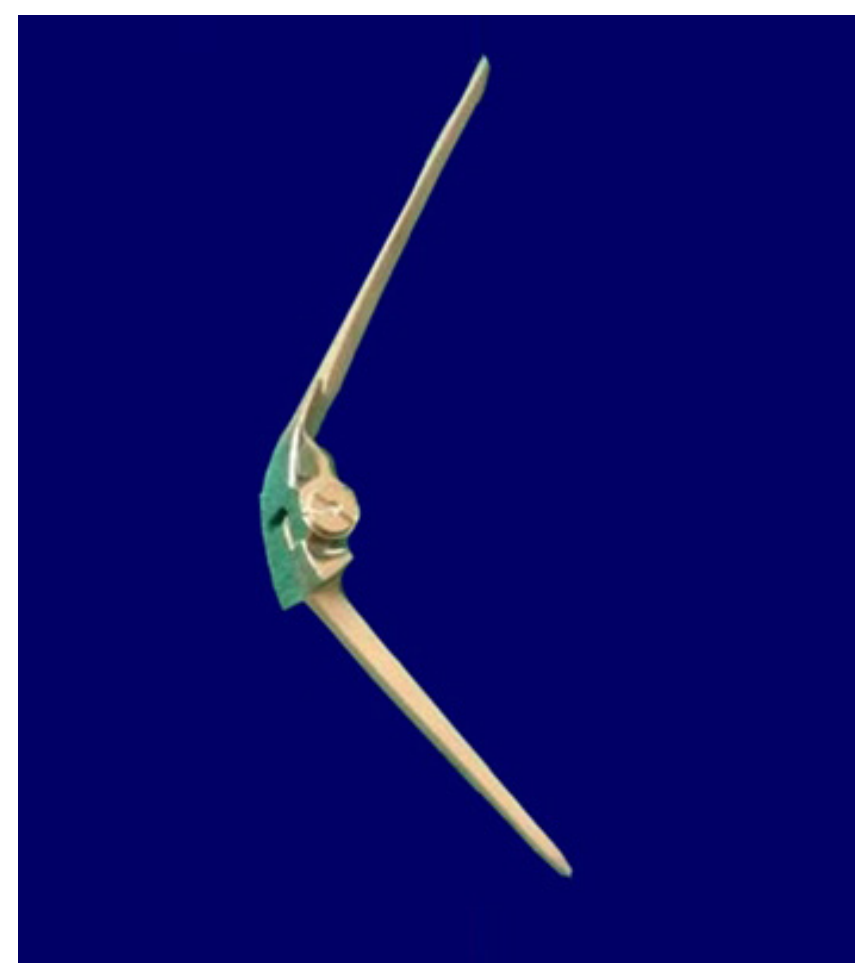

Fig. 3. Assembled Baksi prosthesis showing humeral and ulnar components hinged with the main screw and the additional locking screw.

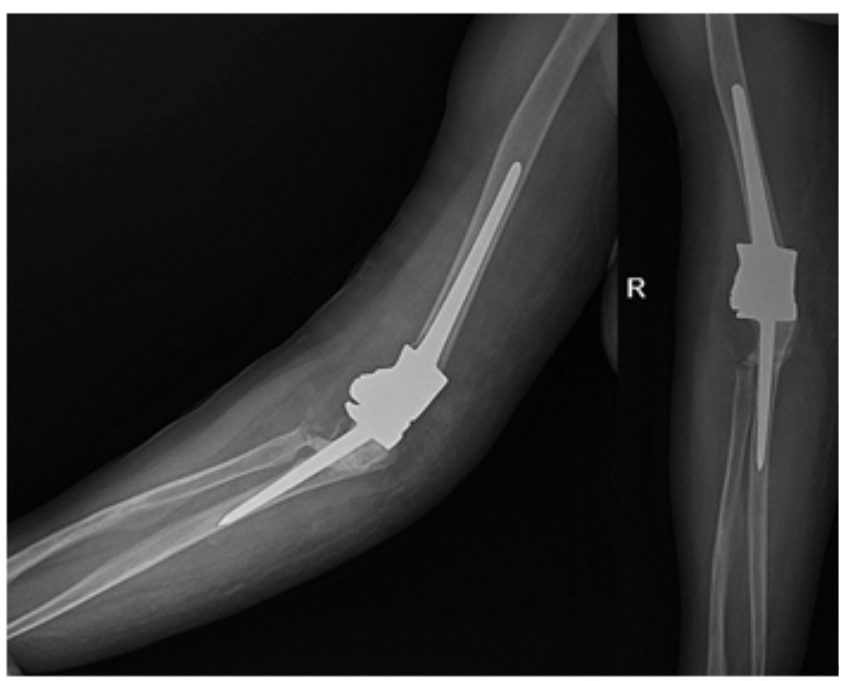

Fig. 4. Immediate postoperative X-ray after total elbow replacement.

such as debridement, synovectomy, and arthroplasty, when needed [1]. The final functional outcome depends on the stage at which the diagnosis was made. When diagnosed early and treated with ATT, results for osteoarticular TB diagnosed can achieve near-normal joint function. However, the result of advanced osteoarticular TB is usually poor [1]. An osteoarticular TB with bony/fibrous ankylosis that has healed can be treated with ar-

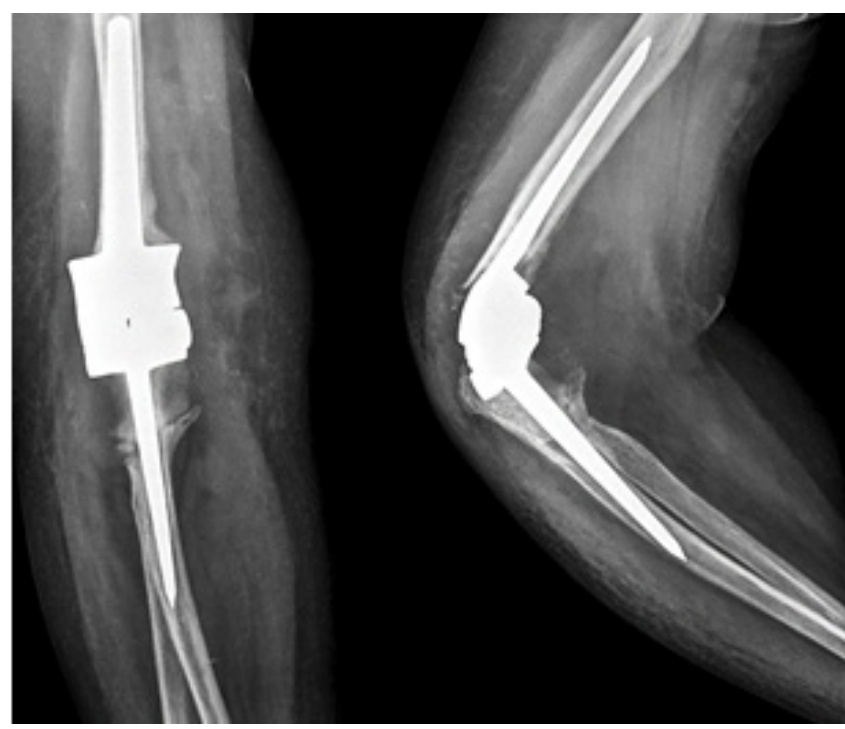

Fig. 5. Follow-up X-ray 3 years after surgery with no signs of loosening.

throplasty to improve the range of movements. Guidance for achieving early and good functional outcomes has been described for treating active advanced articular TB of joints, including the hip and knee, with arthroplasty [4]. Guidelines for ATT preoperatively are controversial and there is currently no consensus and the approach can vary from no preoperative ATT to 2 weeks to 3 months preoperative ATT [5]. Most authors, however, advocate for preoperative ATT to avoid the risk of reactivation [5]. The following measures have been outlined to avoid intra-operative joint reactivation: (1) thorough debridement and synovectomy, (2) complete excision of the sinus tract, if present, and (3) complete removal of the necrotic bone $[4,6]$.

Single-stage or two-stage arthroplasties can be performed, however, unlike other bacterial infections, single-stage arthroplasty does not lead to periprosthetic joint infections for several reasons. First, Mycobacterium tuberculosis (MTB) divides every 15 to 20 hours, which is extremely slow compared with other bacteria [6]. In addition, periprosthetic infections are primarily caused by common biofilm-forming bacteria. MTB rarely adheres to metal surfaces and has little or no biofilm formation potential [7]. As an example, spinal implants are used routinely before MTB is eradicated. Further, two-stage arthroplasty has only been advised in the presence of the sinus tract [4].

Although this case did not experience reactivation in the 3 years of follow-up, we recommend preoperative ATT before arthroplasty. We did not use preoperative ATT since the diagnosis was retrospective, therefore, based on this retrospective analysis, important insights for future cases can be noted. Synovial biopsy and histopathology have been considered to have a diagnosis rate 

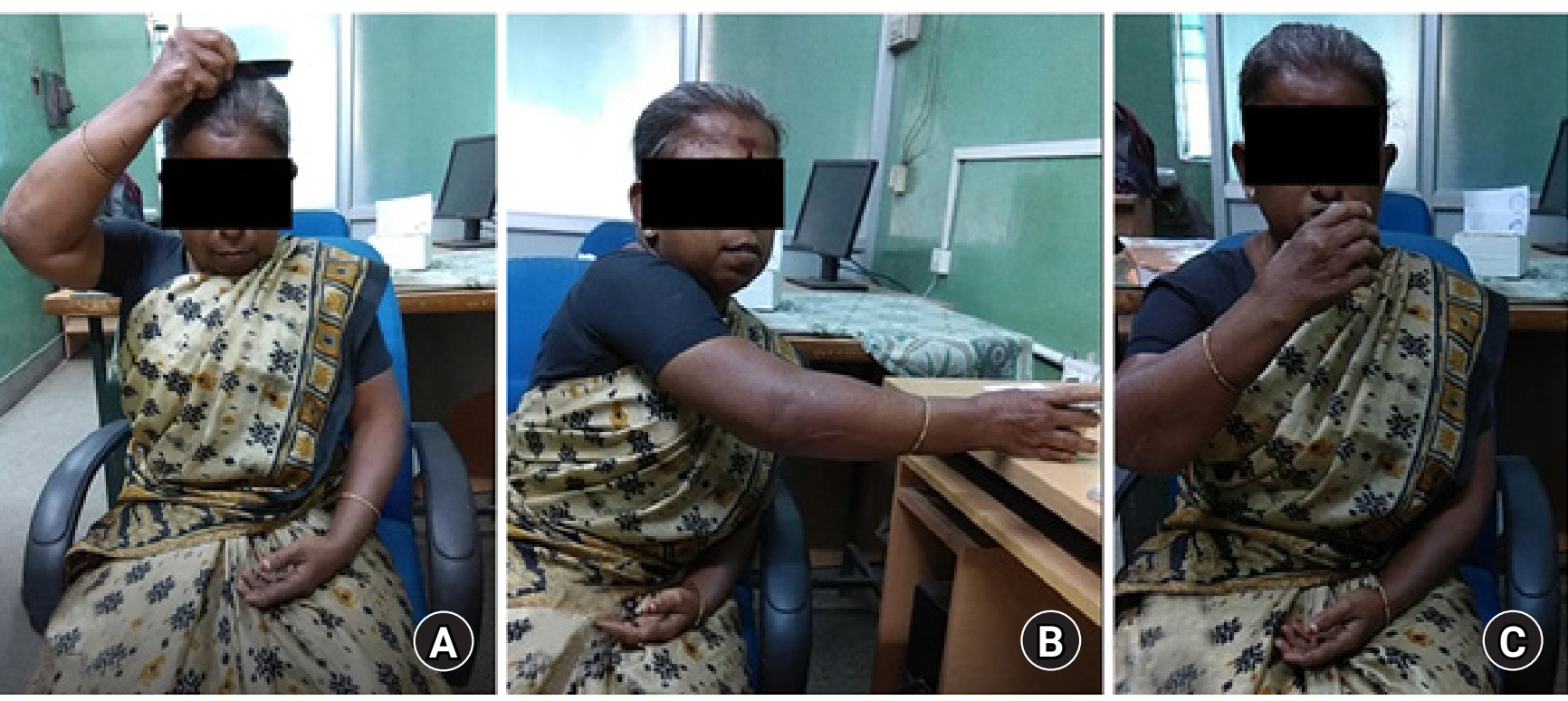

Fig. 6. Follow-up images demonstrating the patient's range of movements postoperatively. The patient reported that she was able to comfortably comb (A), stretch to lift a tumbler (B), and drink water using the affected side (C).

of $72 \%$ to $97 \%$ in osteoarticular TB. A study by Jain et al. [8] reported a $100 \%$ positivity rate from fine-needle aspiration and histopathology. Arthroscopic synovial biopsy is considered to be the best method because the lesion is visualized before the biopsy is taken [9]. However, in endemic countries, even after a negative biopsy, the possibility of TB should not be ruled out. This conclusion is further strengthened by the study of Bae et al. [10], which analyzed 227 biopsy specimens and concluded that in areas of high TB prevalence, TB should not be ruled out even if the biopsy specimen does not show classical granulomatous inflammation or caseous necrosis. We did not perform an open biopsy after the negative first arthroscopic biopsy because arthroscopic biopsy sample adequacy. Further, the diagnosis is particularly challenging in atypical presentations without any pre-existing TB lesions (primary TB). Therefore, recommendations for clinicians that assess future cases are to: (1) include a bone biopsy when performing an arthroscopic synovial biopsy (the combined biopsy might increase the positivity rate) and (2) discuss similar rare presentations in clinic-pathological meetings and seek guidance for further recommendations.

The elbow joint is a small joint and during TEA, a relatively large amount of bone is removed for prosthesis placement. This radical debridement may contribute to reducing the chance of reactivation. Hence, active advanced articular TB in joints like the elbow might be a good candidate for successful early TEA. This will help patients return to their routines and improve their quality of life. Additional studies are needed to confirm these findings with similar approaches in patients. Atypical presenta- tions of osteoarticular TB such as primary TB of the elbow joint pose a challenge in diagnosis. In endemic areas, clinicians should be cautious before excluding such a diagnosis, even after a negative biopsy. Future research should investigate whether active TB of small joints, like the elbow can be treated with ATT and early arthroplasty.

\section{ORCID}

Radhakrishnan Pattu https://orcid.org/0000-0002-0526-1117 Girinivasan Chellamuthu https://orcid.org/0000-0001-5800-714X Kumar Sellappan https://orcid.org/0000-0002-7094-5005 Kamalanathan Chendrayan https://orcid.org/0000-0003-4250-6379

\section{REFERENCES}

1. Lin YM, Tan TS, Lee TS. Tuberculous synovitis of the elbow joint. J Formos Med Assoc 2001;100:568-70.

2. Dhillon MS, Goel A, Prabhakar S, Aggarwal S, Bachhal V. Tuberculosis of the elbow: a clinicoradiological analysis. Indian J Orthop 2012;46:200-5.

3. Baksi DP. Sloppy hinge prosthetic elbow replacement for post-traumatic ankylosis or instability. J Bone Joint Surg Br 1998;80:614-9.

4. Kim YY, Ahn JY, Sung YB, et al. Long-term results of Charnley low-friction arthroplasty in tuberculosis of the hip. J Arthroplasty 2001;16(8 Suppl 1):106-10.

5. Wang Q, Shen H, Jiang Y, et al. Cementless total hip arthroplas- 
ty for the treatment of advanced tuberculosis of the hip. Orthopedics 2011;34:90.

6. Zhang YC, Zhang H. One-stage total joint arthroplasty for patients with active tuberculosis. Orthopedics 2013;36:328-30.

7. Ma J, Li GQ, Cao L. Adhesive ability of Mycobacterium tuberculosis onto the surface of different joint prosthesis materials. Chin J Tissue Eng Res 2012;16:8807-12.

8. Jain AK, Jena SK, Singh MP, Dhammi IK, Ramachadran VG, Dev G. Evaluation of clinico-radiological, bacteriological, sero- logical, molecular and histological diagnosis of osteoarticular tuberculosis. Indian J Orthop 2008;42:173-7.

9. Singhal O, Kaur V, Kalhan S, Singhal MK, Gupta A, Machave Y. Arthroscopic synovial biopsy in definitive diagnosis of joint diseases: an evaluation of efficacy and precision. Int J Appl Basic Med Res 2012;2:102-6.

10. Bae KM, Lim SC, Kim HH, et al. The relevance of biopsy in tuberculosis patients without human immunodeficiency virus infection. Am J Trop Med Hyg 2015;92:636-40. 